\title{
Valoración y evaluación por parte de pedagogos en formación respecto de las competencias específicas del Grado de Pedagogía de la Universidad de Granada
}

\author{
Assessment and evaluation by pedagogues in training regarding \\ the specific competences of the Degree in Pedagogy at the University of Granada
}

\section{Carmen del Pilar Gallardo-Montes ${ }^{a}$, María Jesús Caurcel Cara ${ }^{b}$, Antonio Rodríguez Fuentes ${ }^{a}$}

${ }^{a}$ Departamento de Didáctica y Organización Escolar,

Facultad de Ciencias de la Educación de la Universidad de Granada, España.

cgallardo@ugr.es, arfuente@ugr.es

${ }^{b}$ Departamento de Psicología Evolutiva y de la Educación, Universidad de Granada, España. caurcel@ugr.es

\section{RESUMEN}

El Proceso Bolonia supuso la implantación de competencias en los grados universitarios, las cuales determinan qué habilidades tienen que alcanzar el estudiantado en relación con su perfil profesional. Se ha seguido un enfoque cuantitativo, con un diseño no experimental, descriptivo y trasversal con la finalidad de valorar el grado de desarrollo, dominio y relevancia que los estudiantes tienen sobre las competencias específicas. Se administró un cuestionario ad hoc a 134 estudiantes de $3^{\circ}$ y $4^{\circ}$ curso, realizándose análisis descriptivos (media y desviación típica) e inferenciales no paramétricos (pruebas de Wilcoxon, U Mann-Whitney y Kruskal-Wallis). Los participantes les otorgaron una relevancia notable a las competencias específicas para el desempeño de la profesión; sin embargo, existe desequilibrio entre el desarrollo de las mismas a lo largo de la formación y el dominio alcanzado, siendo necesario potenciar ambos aspectos. Del mismo modo, se aprecian diferencias significativas en torno a las tres variables planteadas.

Palabras claves: Competencias específicas, Educación Superior, Pedagogía, Educación basada en competencias, Evaluación de estudiantes.

\footnotetext{
ABSTRACT

The Bologna Process involved the introduction of competencies in university degrees, which determine what skills the students have to achieve in relation to their professional profile. A quantitative approach has been followed, with a non-experimental, descriptive and cross-sectional design in order to assess the degree of development, mastery and relevance that students have over specific competences. An ad hoc questionnaire was administered to 134 3rd and 4th grade students, and descriptive analyzes (mean and standard deviation) and non-parametric inferential analyzes were performed (Wilcoxon, U Mann-Whitney and Kruskal-Wallis tests). The participants gave a remarkable relevance to the specific competences for the performance of the profession; However, there is an imbalance between the development of the same throughout the training and the domain achieved, being necessary to enhance both aspects. Similarly, there are significant differences around the three variables proposed.

Key words: Specific competences, Higher Education, Pedagogy, Competency-based education, Student evaluation.
} 
Estudios Pedagógicos XLVII N 2: 197-213, 2021

VALORACIÓN Y EVALUACIÓN POR PARTE DE PEDAGOGOS EN FORMACIÓN RESPECTO DE LAS COMPETENCIAS ESPECÍFICAS DEL GRADO DE PEDAGOGÍA DE LA UNIVERSIDAD DE GRANADA

\section{INTRODUCCIÓN}

Con la implantación del Proceso Bolonia en las universidades españolas, en el año 1999, se plantea un modelo educativo universitario en el cual convergen los estudios superiores de toda Europa (Caurcel et al., 2008). De este modo, el objetivo planteado por el Espacio Europeo de Educación Superior (EEES) promueve que para el año 2010, y en adelante, la movilidad entre alumnado de distintas universidades europeas, la empleabilidad y el reconocimiento de grados cursados en toda Europa sea un proceso posible y completamente implantado. Para ello, se desarrollan una serie de competencias acordes a cada titulación universitaria, la cuales se formulan atendiendo a unos perfiles profesionales elaborados de acuerdo a una serie de competencias genéricas, transversales y específicas. Para Castillo y Cabrerizo (2010) el concepto de competencia es claro:

Una competencia es...la capacidad de aplicar conocimientos -lo que se sabe- junto con las destrezas y habilidades -lo que se sabe hacer- para desempeñar una actividad profesional, de manera satisfactoria y en un contexto determinado, de manera satisfactoria -sabiendo ser- uno mismo y sabiendo estar con los demás. (p. 64).

La formación basada en competencias tiene como eje principal facilitar al estudiante herramientas que le permitan desenvolverse en aspectos prácticos, ya que las competencias van a suponer ese conjunto de saberes saber hacer, saber ser, saber convivir y saber conocer (Rodríguez, 2007). En definitiva, en el contexto universitario en el que nos situamos actualmente, se encuentra un currículum académico relacionado íntimamente con las competencias (García y Bernabé, 2019). En esta línea, la OCDE (2005) propone la siguiente definición: "Una competencia es más que conocimientos y destrezas. Implica la capacidad para responder a demandas complejas, utilizando y movilizando recursos psicosociales (incluyendo habilidades y actitudes) en un contexto particular" (p. 3).

La manera de transmitir conocimientos o, mejor dicho, el proceso de enseñanzaaprendizaje ha cambiado con los años ya que las exigencias sociales también lo han hecho, lo que ha supuesto un reajuste de todo lo preestablecido. No solo es relevante educar y guiar al estudiantado según unas competencias esenciales, sino que éstas deben estar en consonancia con el desarrollo por y para la sociedad.

Como menciona Martí et al. (2018) "los cambios en la dinámica entre docenteestudiante, la necesidad de adaptación a ambientes globales y la aparición de nuevas tecnologías, han presionado cambios en el modelo educativo acerca de la formación del pensamiento crítico y de la autonomía moral" (p. 49). De la misma forma, el rol del docente y el discente ha cambiado, siendo las metodologías, la manera de investigar y las fuentes de conocimiento diversas. Consecuentemente, las titulaciones universitarias deben estar al día y proporcionar precisamente esas competencias a una juventud y a una sociedad que se nutre diariamente de distintos ámbitos sociales. En este sentido, son varios los autores que han orientado sus estudios en torno a esta temática (Coiduras et al., 2018; Flores-Lueg y Roig, 2018; García et al., 2014; López et al., 2016; Makuc, 2018; Pérez et al., 2019; Reche et al., 2019; Richart et al., 2019; Serrano y Pontes, 2015; Velásquez et al., 2018). 


\subsection{JUSTIFICACIÓN}

El grado de Pedagogía de la Universidad de Granada (España) pretende formar profesionales en instituciones, contextos, recursos y procesos educativos, formativos, de desarrollo personal, profesional, social y cultural que concurren de forma integrada en las personas y grupos a lo largo del ciclo vital. La titulación de Pedagogía difiere de los grados en Maestro de Educación Primaria y de Educación Infantil, pues su labor no se limita a la intervención en el ámbito escolar sino que abarca otros campos, como el cultural, social o empresarial. Dentro del escolar sus funciones difieren y se relacionan con la atención a las necesidades educativas, la orientación vocacional/educativa y la asesoría a docentes y familias.

Dado que este estudio se centra en conocer el nivel competencial del alumnado del grado de Pedagogía de la Universidad de Granada, es preciso concretar cuáles son las competencias propuestas. Así, a partir de lo recogido en el Libro Blanco: Título de Grado en Pedagogía y Educación Social (ANECA, 2005) -31 competencias específicas-, de lo propuesto en el Acuerdo de la Comisión Permanente de la Conferencia de Decanos y Directores de Magisterio y Educación -en la sesión de 6 de marzo de 2008- y en los acuerdos finales adoptados por la Comisión de la Titulación de Pedagogía formada por las Universidades de Sevilla, Málaga y Granada, -reunida en la Universidad de Sevilla, el 24 de junio de 2008-, se seleccionaron las siguientes competencias específicas:

C1: Conocer y comprender las bases teóricas y epistemológicas de los procesos y acciones formativas y educativas.

C2: Conocer y analizar las organizaciones, instituciones y sistemas de educación y formación como productos culturales e históricos y su influencia en la política y legislación educativa nacional e internacional.

C3: Comprender las bases del desarrollo personal, social y cultural y su incidencia en el proceso educativo.

C4: Organizar, gestionar y evaluar centros, instituciones, servicios y sistemas educativos y formativos.

C5: Diseñar, desarrollar, gestionar y evaluar planes, proyectos, programas, recursos y materiales para la acción educativa y/o formativa en distintos ámbitos y contextos.

C6: Diseñar, ejecutar y evaluar planes de formación del profesorado, de formadores y de otros profesionales en diferentes contextos.

C7: Diagnosticar, orientar y asesorar a personas, colectivos e instituciones en ámbitos educativos y formativos.

C8: Conocer y comprender las bases teóricas y epistemológicas de la investigación educativa.

C9: Identificar, analizar y aplicar los procedimientos de la investigación educativa para emitir juicios argumentados que permitan la mejora de la práctica educativa.

C10: Asesorar en la toma de decisiones sobre problemas relevantes.

C11: Conocer, analizar y atender a la diversidad social, educativa y cultural por razón de género, clase, etnia, edad, discapacidad, religión u otras.

C12: Promover, planificar y gestionar la implantación de procesos de innovación educativa y de modelos de gestión de la calidad.

C13: Realizar estudios prospectivos y evaluativos sobre características, necesidades y demandas pedagógicas. 
Estas competencias están presentes, en mayor o menor medida, en el Grado de Pedagogía, vertebradas en cada una de las distintas asignaturas planteadas. Dichas competencias se desarrollan con la finalidad de vislumbrar qué necesita el alumnado para obtener el graduado actual en Pedagogía, en tanto que son formuladas atendiendo a aspectos esenciales que el estudiante tiene que alcanzar y perfeccionar a lo largo de su periplo universitario.

\subsection{ANTECEDENTES}

Son diversos los estudios previos que han analizado las competencias y su desarrollo en el ámbito de la Educación Superior. Así, Ruiz (2010) evalúa el desarrollo de las competencias genéricas y específicas en la asignatura "Estrategias en las adaptaciones curriculares" de Pedagogía (Universidad Complutense de Madrid, España). De este modo, se recoge que competencias como "Capacidad de análisis y síntesis", "Toma de decisiones" o "Capacidad para empatizar", se encuentran en un nivel alto de ejecución, por lo que se encuentran ya consolidadas. Por otro lado, en un nivel medio de valoración, desarrollo y ejecución están las competencias "Capacidad para generar nuevas ideas", "Planificación y gestión del tiempo", "Trabajo en equipo", "Comunicación con personas no expertas en la materia" y "Habilidad para trabajar con autonomía".

Para la titulación de Psicología, en la Universidad de Huelva (España), Alonso-Martín (2010) compara la valoración que los estudiantes hacen de las competencias transversales al inicio y al final del curso. Se halla que las competencias más valoradas son las de "Análisis y síntesis" y "Capacidad de autocrítica". En cambio, competencias como "Capacidad de liderazgo", "Iniciativa y espíritu emprendedor" o "Capacidad de trabajo en equipo" son a las que menos importancia les otorgan los estudiantes de cara al mercado laboral. De este modo, les conceden más relevancia a las competencias vinculadas a lo cognitivo.

Por otro lado, Pérez et al. (2013) se centran en la valoración de las competencias básicas, transversales, generales y específicas en el grado de Educación Social (Universidad de Murcia, España). Los resultados evidencian el buen dominio de la competencia relacionada con las "Habilidades de trabajo autónomo" y el bajo dominio y desarrollo de la "Transmisión de conocimientos y soluciones". En relación con las competencias específicas, las mejores puntuaciones en cuanto al dominio y el desarrollo las obtienen la "Elaboración de proyectos de intervención" y el "Sentido ético y social de la profesión", y la más baja, la competencia relacionada con el "Trabajo con instituciones educativas y sociales".

Continuando en la línea de los estudios anteriores, González y Martínez (2015) realizan una comparación entre el desarrollo, la utilidad y la relevancia de las competencias transversales entre la Universidad de Murcia (UM, España) y la Universidad Católica de Córdoba (UCC, Argentina). Obteniendo los estudiantes de la UCC mejores resultados en cuanto al nivel de desarrollo de las competencias transversales; mientras que los estudiantes de la UM arrojan puntuaciones mayores en cuanto a la utilidad que le confieren a las competencias.

Como suma a lo anterior, González et al. (2015) analizan la percepción del alumnado de Pedagogía de la Universidad de Murcia sobre las competencias básicas, transversales, generales y específicas en cuanto a su desarrollo y dominio. El alumnado no percibe ninguna de las competencias con un nivel alto de desarrollo y dominio. De manera 
general, las medias obtenidas muestran un nivel de desarrollo y dominio suficiente para las competencias básicas y generales, notable para las transversales y aceptable para las específicas. Tras el análisis, concluyen que existe un desequilibrio entre lo que las competencias plantean y lo que el estudiantado consigue aprender durante su formación. Por tanto, es necesario reforzar el desarrollo de las competencias en aula universitaria, así como su dominio entre los estudiantes.

Por otro lado, en relación con estudios que reflejen diferencias significativas según la edad o el sexo Molero, Ortega y Moreno (2010) en su investigación sobre las diferencias en la adquisición de competencias emocionales en función del género, concluyen que existen diferencias significativas en dos subescalas: "Percepción o Atención a los sentimientos" a favor de las mujeres, y "Regulación o Reparación de las emociones" a favor de los hombres.

A su vez, Martín et al. (2012) han indagado acerca de qué métodos docentes promueven mejor la adquisición de competencias en sus estudiantes. Los autores no aprecian diferencias significativas en relación con la edad, aunque sí respecto del sexo, siendo las mujeres las que aportan valoraciones más altas en la mayoría de los ítems en comparación con los hombres.

En ese mismo año se encuentra el trabajo de Aguilar et al. (2015), relacionado con la evaluación de las competencias del profesorado. Del análisis de datos se extraen diferencias en función del sexo y de la edad en el nivel competencial vinculado a la acción tutorial. Los hombres arrojaban medias superiores sobre el nivel competencial respecto a la acción tutorial. Con respecto a la edad, los participantes más jóvenes presentaban medias más altas en cuanto a las competencias evaluadas.

Por su parte, Díaz et al. (2016) en su investigación sobre las competencias TIC de estudiantes universitarios del ámbito de la educación y su relación con las estrategias de aprendizaje, hallan diferencias en función de dos variables principales: el sexo y la edad del alumnado. Las mujeres presentan mejor nivel en los aspectos afectivos, metacognitivos o motivacionales; resaltando los hombres, sin embargo, en estrategias superficiales. En cuanto a la edad, se observa una tendencia inversa en la mayor parte de las dimensiones de la competencia tecnológica, donde el grupo tiene menor nivel competencial según aumenta la edad. Y la competencia ética no presenta influencia significativa según la edad.

Un trabajo más reciente es el realizado por Rodríguez et al. (2018), en el que evalúan las competencias básicas de los estudiantes universitarios en torno a los procesos de e-evaluación orientada al aprendizaje. Se aprecian diferencias significativas en cuanto al sexo en ocho de las diez competencias valoradas, a favor de las mujeres. En contraposición, los hombres demuestran mayor nivel competencial en tareas relacionadas con las competencias "argumentación" y "resolución de problemas".

\subsection{OBJETIVOS}

Atendiendo a la revisión teórica realizada, este estudio pretende:

1) Conocer el nivel competencial alcanzado por el alumnado del Grado de Pedagogía de la Universidad de Granada en relación con el desarrollo, dominio y relevancia de las competencias específicas designadas para dicha titulación.

2) Analizar si existen diferencias entre las valoraciones que hacen los estudiantes en función del sexo, la edad y la nota media. 


\section{DISEÑO Y MÉTODO}

Dado que el propósito de esta investigación es conocer la valoración que hace el alumnado con respecto al desarrollo, dominio y relevancia, el diseño utilizado atiende a un enfoque cuantitativo. El diseño es no experimental, ya que no se introducen modificaciones en las variables, sino descriptivo y transversal, puesto que los datos se recogieron en un único momento (Montero y León, 2007).

\subsection{PARTICIPANTES}

La muestra estuvo conformada por estudiantes de $3^{\circ}$ y $4^{\circ}$ curso del grado de Pedagogía de la Universidad de Granada. El alumnado de $1^{\circ}$ y $2^{\circ}$ ha sido excluido por su menor experiencia y conocimiento de la titulación. Para su selección se ha realizado un muestreo probabilístico aleatorio simple (Pérez, 2005). Durante el curso académico 2017-2018, una población de 548 alumnos estaba matriculados en el grado de Pedagogía en la Universidad de Granada (Memoria Académica, 2017-2018); de los cuales 244 cursaban $3^{\circ}$ y $4^{\circ}$ curso, excluyendo al alumnado perteneciente a los programas de intercambio ERASMUS y SICUE (Sistema Integral de Gestión de Alumnos [SIGA], 2017-2018). La muestra de esta investigación es de 134 estudiantes, suficientemente representativa con un nivel de confianza de $95 \%$ y un error muestral de $5 \%(\alpha=, 05+5 \%)$.

El rango de edad de los participantes oscila entre los 20 y los 28 años $(M=21,95$ años, $D T=1,56), 112$ son mujeres $(83,58 \%)$ y 22 hombres $(16,42 \%)$, no encontrándose balanceada por sexo, dado el predominio de las mujeres en los estudios relacionados con las Ciencias de la Educación, en general, y en la titulación, en particular. El 97\% afirma que asiste a las clases de manera habitual -algunas veces (3\%) - y la calificación obtenida, por la mayoría, a lo largo del grado es de notable $(97 \%)$-aprobado $(2,2 \%)$ y sobresaliente $(0,7 \%)$ -

\subsection{INSTRUMENTO}

Para poder medir las competencias específicas planteadas, y tomando como referencia el "Cuestionario de Evaluación de Competencias de Grado en Pedagogía (CECGRAP)" elaborado para la Universidad de Murcia por González et al. (2015), se ha diseñado un cuestionario ad hoc, denominado "Cuestionario de evaluación de competencias específicas de los estudiantes del Grado de Pedagogía”. Existen otros instrumentos similares, como el diseñado y validado por Romero y Faouzi (2018) para medir competencias pedagógicas para maestros en ejercicio o para medir competencias específicas para maestros especialistas en formación (Domingo et al., 2010; Gallego y Rodríguez, 2015), empero ninguno respondía concretamente a los objetivos de la presente investigación.

El cuestionario consta de dos apartados. El primero, sobre datos sociodemográficos del estudiante: sexo, edad, asistencia a clase y nota media. Y el segundo en el cual se demanda la autovaloración de las 13 competencias específicas del Grado, en cuanto al desarrollo de dicha competencia a lo largo del Grado; dominio o agilidad desempeñada en cada una de ellas; y relevancia e importancia que se le otorga a cada competencia en la carrera y de cara al mundo laboral (Cfr. Tabla 1).

Se ha utilizado una escala de respuesta tipo Likert ( $1=$ Nada, $2=$ Casi nada, $3=\mathrm{A}$ veces, $4=$ A menudo, $5=$ Bastante y $6=$ Mucho). Para la interpretación de las valoraciones 
hay que tener en cuenta los siguientes criterios de interpretación de la escala utilizada: cuando la media obtenida sea menor a 3 indica que no se ha alcanzado el nivel óptimo de desarrollo y dominio de esa competencia o que no es relevante; entre 3-3.9, se considera suficiente, aunque mejorable; entre 4-4.9, notable y, por último, se ha alcanzado un alto nivel cuando las medias estén entre 5-6.

Tabla 1. Competencias específicas pedagógicas sometidas a autoevaluación tipo Likert (de 1 a 6) del alumnado, según desarrollo, dominio y relevancia

\begin{tabular}{|c|c|c|c|}
\hline Competencia & Desarrollo & Dominio & Relevancia \\
\hline $\begin{array}{l}\text { 1. Conocer y comprender las bases teóricas y } \\
\text { epistemológicas de los procesos y acciones } \\
\text { formativas y educativas. }\end{array}$ & 123456 & 123456 & 123456 \\
\hline $\begin{array}{l}\text { 2. Conocer y analizar las organizaciones, instituciones } \\
\text { y sistemas de educación y formación como } \\
\text { productos culturales e históricos y su influencia } \\
\text { en la política y legislación educativa nacional e } \\
\text { internacional. }\end{array}$ & 123456 & 123456 & 123456 \\
\hline $\begin{array}{l}\text { 3. Comprender las bases del desarrollo personal, } \\
\text { social y cultural y su incidencia en el proceso } \\
\text { educativo. }\end{array}$ & 123456 & 123456 & 123456 \\
\hline $\begin{array}{l}\text { 4. Organizar, gestionary evaluar centros, instituciones, } \\
\text { servicios y sistemas educativos y formativos. }\end{array}$ & 123456 & 123456 & 123456 \\
\hline $\begin{array}{l}\text { 5. Diseñar, desarrollar, gestionar y evaluar planes, } \\
\text { proyectos, programas, recursos y materiales para la } \\
\text { acción educativa y/o formativa en distintos ámbitos } \\
\text { y contextos. }\end{array}$ & 123456 & 123456 & 123456 \\
\hline $\begin{array}{l}\text { 6. Diseñar, ejecutar y evaluar planes de formación del } \\
\text { profesorado, de formadores y de otros profesionales } \\
\text { en diferentes contextos. }\end{array}$ & 123456 & 123456 & 123456 \\
\hline $\begin{array}{l}\text { 7. Diagnosticar, orientar y asesorar a personas, } \\
\text { colectivos e instituciones en ámbitos educativos y } \\
\text { formativos. }\end{array}$ & 123456 & 123456 & 123456 \\
\hline $\begin{array}{l}\text { 8. Conocer y comprender las bases teóricas y } \\
\text { epistemológicas de la investigación educativa. }\end{array}$ & 123456 & 123456 & 123456 \\
\hline $\begin{array}{l}\text { 9. Identificar, analizar y aplicar los procedimientos } \\
\text { de la investigación educativa para emitir juicios } \\
\text { argumentados que permitan la mejora de la práctica } \\
\text { educativa. }\end{array}$ & 123456 & 123456 & 123456 \\
\hline $\begin{array}{l}\text { 10. Asesorar en la toma de decisiones sobre problemas } \\
\text { relevantes. }\end{array}$ & 123456 & 123456 & 123456 \\
\hline
\end{tabular}




\begin{tabular}{|l|l|l|l|}
\hline $\begin{array}{l}\text { 11. Conocer, analizar y atender a la diversidad social, } \\
\text { educativa y cultural por razón de género, clase, } \\
\text { etnia, edad, discapacidad, religión u otras. }\end{array}$ & 123456 & 123456 & 123456 \\
\hline $\begin{array}{l}\text { 12. Promover, planificar y gestionar la implantación de } \\
\text { procesos de innovación educativa y de modelos de } \\
\text { gestión de la calidad. }\end{array}$ & 123456 & 123456 & 123456 \\
\hline $\begin{array}{l}\text { 13. Realizar estudios prospectivos y evaluativos } \\
\text { sobre características, necesidades y demandas } \\
\text { pedagógicas. }\end{array}$ & 123456 & 123456 & 123456 \\
\hline
\end{tabular}

Fuente: elaboración propia.

\subsection{PROCEDIMIENTO}

Este estudio comienza con una revisión bibliográfica para plantear el estado de la cuestión y concretar los objetivos de investigación. A su vez, fueron revisados los instrumentos de recogida de información, no encontrando ninguno que midiese y valorase directamente las competencias específicas en el Grado de Pedagogía de la Universidad de Granada, por lo que se procedió con el diseño ad hoc de uno adecuado al tema en cuestión. Para garantizar su validez, se realizó un proceso de validación de contenido de tipo cualitativo mediante juicio de expertos, con investigadores de distintas especialidades y grupos de investigación. Para la administración del cuestionario, se ha contactado con los docentes de las asignaturas para acordar los horarios y contextos más adecuados para la cumplimentación del mismo. Se ha explicado al estudiantado los objetivos de la investigación, el contenido del cuestionario y el carácter anónimo, voluntario y confidencial de los datos. Seguidamente, se ha procedido a la recogida de los datos, para lo que se dispuso de 15 minutos para cumplimentar el cuestionario.

\subsection{ANÁLISIS DE DATOS}

Los datos han sido tratados con el paquete estadístico SPSS versión 24.0 para Windows. Para dar respuesta a los objetivos planteados se han utilizado estadísticos descriptivos porcentuales, de tendencia central (media) y dispersión (desviación típica), así como inferenciales no paramétricos -dado que los datos no presentan una distribución normalpara detectar las diferencias significativas entre las variables estudiadas. Se ha utilizado la prueba de Wilcoxon para comparar las medias relacionadas respecto al desarrollo, dominio y relevancia y determinar la existencia de relaciones o diferencias entre éstas. Por otro lado, también, se utiliza la prueba de Mann-Whitney para determinar si existen o no diferencias significativas entre dos muestras independientes (hombres y mujeres) y la prueba de Kruskal-Wallis para la comparación de los datos obtenidos en cuanto a la edad y en cuanto a la nota media. Para analizar los datos resultantes en relación con la edad, se establecieron tres grupos: Grupo 1 de 20-21 años, Grupo 2 de 22-23 años y Grupo 3 de 24 años en adelante. En referencia a la nota media, se establecieron de igual modo tres grupos: Aprobado, Notable y Sobresaliente. 


\section{RESULTADOS}

Respecto al grado de desarrollo (Cfr. Tabla 2), observamos que el 53,84\% de las competencias se encuentran en un nivel de desarrollo notable, el 46,16\% suficiente y que ninguna ha sido valorada con un alto nivel. Las competencias específicas que los participantes consideran que más han desarrollado a lo largo de la titulación son las de: "Diseñar, desarrollar, gestionar y evaluar planes, proyectos, programas, recursos y materiales para la acción educativa y/o formativa en distintos ámbitos y contextos" (CDE5) y "Conocer, analizar y atender a la diversidad social, educativa y cultural por razón de género, clase, etnia, edad, discapacidad, religión u otras" (CDE11), ambas con una media de 4,31. Las competencias menos desarrolladas han sido dos: "Asesorar en la toma de decisiones sobre problemas relevantes" (CDE10) y "Realizar estudios prospectivos y evaluativos sobre características, necesidades y demandas pedagógicas" (CDE13), con una valoración media de 3,91.

En cuando al dominio (Cfr. Tabla 2), el 61,53\% de las competencias se encuentran en un nivel suficiente, el $38,47 \%$ notable y ninguna se valora con un alto nivel. Las competencias que más dominan son: "Diseñar, desarrollar, gestionar y evaluar planes, proyectos, programas, recursos y materiales para la acción educativa y/o formativa en distintos ámbitos y contextos" (CDO5) y "Conocer, analizar y atender a la diversidad cultural por razón de género, clase, etnia, edad, discapacidad, religión u otras" (CDO11) con valores de 4,15 y 4,33, respectivamente. En cambio, las que menos dominan son "Organizar, gestionar y evaluar centros, instituciones, servicios y sistemas educativos y formativos" (CDO4) (M = $3,69)$ y "Realizar estudios prospectivos y evaluativos sobre características, necesidades y demandas pedagógicas" (CDO13) $(\mathrm{M}=3,73)$.

Con relación a la relevancia (Cfr. Tabla 2), se halla que el 92,31\% de las competencias se perciben con una relevancia notable y solo una como alta. Los estudiantes otorgan más relevancia e importancia a: "Conocer, analizar y atender a la diversidad social, educativa y cultural por razón de género, clase, etnia, edad, discapacidad, religión u otras" (CRE11) $(\mathrm{M}=5,12)$, "Diagnosticar, orientar y asesorar a personas, colectivos e instituciones en ámbitos educativos y formativos" (CRE7) ( $\mathrm{M}=4,98)$, y "Asesorar en la toma de decisiones sobre problemas relevantes" (CRE10) $(\mathrm{M}=4,97)$. Sin embargo, otorgan menor importancia a: "Conocer y comprender las bases teóricas y epistemológicas de los procesos y acciones formativas y educativas" (CRE1) $(\mathrm{M}=4,25)$ y "Conocer y analizar las organizaciones, instituciones y sistemas de educación y formación como productos culturales e históricos y su influencia en la política y legislación educativa nacional e internacional" (CRE2) $(\mathrm{M}=4,31)$. 
Estudios Pedagógicos XLVII N² 2: 197-213, 2021

VALORACIÓN Y EVALUACIÓN POR PARTE DE PEDAGOGOS EN FORMACIÓN RESPECTO DE LAS COMPETENCIAS ESPECÍFICAS DEL GRADO DE PEDAGOGÍA DE LA UNIVERSIDAD DE GRANADA

Tabla 2. Medias y dispersión de las competencias en función al desarrollo, dominio y relevancia

\begin{tabular}{|c|c|c|c|c|c|c|}
\hline \multirow{2}{*}{ Competencia } & \multicolumn{2}{|c|}{ Desarrollo (CDE) } & \multicolumn{2}{c|}{ Dominio (CDO) } & \multicolumn{2}{c|}{ Relevancia (CRE) } \\
\cline { 2 - 7 } & $\boldsymbol{M}$ & $\boldsymbol{D} \boldsymbol{T}$ & $\boldsymbol{M}$ & $\boldsymbol{D} \boldsymbol{T}$ & $\boldsymbol{M}$ & $\boldsymbol{D T}$ \\
\hline 1 & 4,20 &, 86 & 3,78 &, 83 & 4,25 &, 96 \\
\hline 2 & 3,98 &, 86 & 3,79 &, 79 & 4,31 & 1,01 \\
\hline 3 & 4,22 &, 90 & 4,14 &, 99 & 4,62 & 1,15 \\
\hline 4 & 3,99 &, 97 & 3,69 &, 95 & 4,56 & 1,3 \\
\hline 5 & 4,31 & 1,11 & 4,15 & 1,02 & 4,80 & 1,07 \\
\hline 6 & 3,98 & 1,02 & 3,81 & 1,13 & 4,68 & 1,09 \\
\hline 7 & 4,14 & 1,12 & 4,04 & 1,08 & 4,98 &, 91 \\
\hline 8 & 4,19 & 1,07 & 3,81 &, 99 & 4,44 & 1,09 \\
\hline 9 & 4,13 &, 97 & 3,89 &, 91 & 4,60 & 1,19 \\
\hline 10 & 3,94 & 1,13 & 3,93 & 1,12 & 4,97 & 1,09 \\
\hline 11 & 4,31 & 1,13 & 4,33 & 1,05 & 5,12 &, 97 \\
\hline 12 & 3,99 & 1,13 & 3,79 & 1,10 & 4,80 & 1,03 \\
\hline 13 & 3,91 &, 97 & 3,73 & 1,11 & 4,76 &, 96 \\
\hline
\end{tabular}

Nota: $C D E=$ Desarrollo de la competencia; $C D O=$ Dominio de la competencia; $C R E=$ Relevancia de la competencia; $M=$ Media; $D T=$ Desviación típica.

Fuente: elaboración propia a partir del análisis de datos.

Los datos de carácter descriptivo analizados señalan que el alumnado no parece otorgar el mismo nivel de desarrollo, dominio y relevancia a cada competencia. En este sentido, se ha profundizado en el estudio comparativo, observando diferencias estadísticamente significativas entre el desarrollo y el dominio de las competencias 1, 2, 4, 5, 8, 9 y 13 (Cfr. Tabla 3), siendo, en todos los casos, según la visión de los estudiantes, el desarrollo de estas competencias superior a su dominio.

Tabla 3. Resultados de la prueba Wilcoxon para el grado de desarrollo y de dominio de las competencias específicas

\begin{tabular}{|c|c|c|c|c|c|c|c|c|c|c|c|c|c|}
\hline & $\mathbf{1}$ & $\mathbf{2}$ & $\mathbf{3}$ & $\mathbf{4}$ & $\mathbf{5}$ & $\mathbf{6}$ & $\mathbf{7}$ & $\mathbf{8}$ & $\mathbf{9}$ & $\mathbf{1 0}$ & $\mathbf{1 1}$ & $\mathbf{1 2}$ & $\mathbf{1 3}$ \\
\hline $\boldsymbol{Z}$ & $-5,33^{\mathrm{a}}$ & $-2,69^{\mathrm{a}}$ & $-1,04^{\mathrm{a}}$ & $-3,76^{\mathrm{a}}$ & $-2,15^{\mathrm{a}}$ & $-1,95^{\mathrm{a}}$ & $-1,26^{\mathrm{a}}$ & $-4,24^{\mathrm{a}}$ & $-2,83^{\mathrm{a}}$ &,$- 16^{\mathrm{b}}$ &,$- 39^{\mathrm{b}}$ & $-1,88^{\mathrm{a}}$ & $-2,50^{\mathrm{a}}$ \\
\hline Sig. &, $000^{* * * *}$ &, $007^{* *}$ &, 298 &, $000^{* * *}$ &, $031^{*}$ &, 051 &, 208 &, $000^{* * *}$ &, $005^{* *}$ &, 873 &, 695 &, 060 &, $012^{*}$ \\
\hline
\end{tabular}

Nota: ${ }^{a}=$ Se basa en rangos positivos; ${ }^{b}=$ Se basa en rangos negativos; $Z=$ resultados de la prueba de Wilcoxon al comparar desarrollo y dominio de las competencias; Sig. = Significación; Estadísticamente significativas: $* p<, 05 * * p<, 01 * * * p<, 001$

Fuente: elaboración propia a partir del análisis de datos. 
Atendiendo a los datos obtenidos, se puede apreciar como en la Tabla 4 existen diferencias estadísticamente significativas $(p<, 05)$ entre la relevancia y el desarrollo de las competencias 1, 2, 3, 4, 5, 8, 9 y 13. La relevancia otorgada a las competencias por parte de los estudiantes es superior al desarrollo de las mismas a lo largo de la titulación.

Tabla 4. Resultados de la prueba Wilcoxon para el grado de relevancia y de desarrollo de las competencias específicas

\begin{tabular}{|c|c|c|c|c|c|c|c|c|c|c|c|c|c|}
\hline & $\mathbf{1}$ & $\mathbf{2}$ & $\mathbf{3}$ & $\mathbf{4}$ & $\mathbf{5}$ & $\mathbf{6}$ & $\mathbf{7}$ & $\mathbf{8}$ & $\mathbf{9}$ & $\mathbf{1 0}$ & $\mathbf{1 1}$ & $\mathbf{1 2}$ & $\mathbf{1 3}$ \\
\hline $\boldsymbol{Z}$ &,,$- 62^{\mathrm{b}}$ & $-2,95^{\mathrm{b}}$ & $-3,56^{\mathrm{b}}$ & $-4,72^{\mathrm{b}}$ & $-4,25^{\mathrm{b}}$ & $-5,52^{\mathrm{b}}$ & $-6,42^{\mathrm{b}}$ & $-2,16^{\mathrm{b}}$ & $-3,93^{\mathrm{b}}$ & $-7,05^{\mathrm{b}}$ & $-6,46^{\mathrm{b}}$ & $-5,94^{\mathrm{b}}$ & $-6,74^{\mathrm{b}}$ \\
\hline Sig. &, 536 & $, 003^{* *}, 000^{* * *}, 000^{* * *}, 000^{* * *}, 000^{* * *}, 000^{* * *}$ &, $031^{*}$ &, $000^{* * *}$ &, $000^{* * * *}$ & $, 000^{* * * *}, 000^{* * *}, 000^{* * *}$ \\
\hline
\end{tabular}

Nota: ${ }^{b}=$ Se basa en rangos negativos; $Z=$ resultados de la prueba de Wilcoxon al comparar desarrollo y relevancia de las competencias; Sig. $=$ Significación; Estadísticamente significativas: ${ }^{*} p<, 05 * * p<, 01{ }^{* * *} p<, 001$

Fuente: elaboración propia a partir del análisis de datos.

Del mismo modo, en cuanto al dominio y la relevancia, se presentan diferencias estadísticamente significativas $(p<, 05)$ (Cfr. Tabla 5) en las 13 competencias específicas valoradas, considerando que tienen una mayor relevancia que el nivel de dominio alcanzado.

Tabla 5. Resultados de la prueba Wilcoxon para el grado de dominio y de relevancia de las competencias específicas

\begin{tabular}{|c|c|c|c|c|c|c|c|c|c|c|c|c|c|}
\hline & $\mathbf{1}$ & $\mathbf{2}$ & $\mathbf{3}$ & $\mathbf{4}$ & $\mathbf{5}$ & $\mathbf{6}$ & $\mathbf{7}$ & $\mathbf{8}$ & $\mathbf{9}$ & $\mathbf{1 0}$ & $\mathbf{1 1}$ & $\mathbf{1 2}$ & $\mathbf{1 3}$ \\
\hline $\boldsymbol{Z}$ & $-4,63^{\mathrm{b}}$ & $-4,97^{\mathrm{b}}$ & $-4,05^{\mathrm{b}}$ & $-6,80^{\mathrm{b}}$ & $-5,13^{\mathrm{b}}$ & $-6,38^{\mathrm{b}}$ & $-7,12^{\mathrm{b}}$ & $-4,98^{\mathrm{b}}$ & $-5,61^{\mathrm{b}}$ & $-7,13^{\mathrm{b}}$ & $-6,37^{\mathrm{b}}$ & $-7,12^{\mathrm{b}}$ & $-7,48^{\mathrm{b}}$ \\
\hline Sig. & $, 000^{* * *}, 000^{* * *}, 000^{* * *}, 000^{* * *}, 000^{* * *}, 000^{* * *}, 000^{* * *}, 000^{* * * *}, 000^{* * *}, 000^{* * *}, 000^{* * *}, 000^{* * *}, 000^{* * * *}$ \\
\hline
\end{tabular}

Nota: ${ }^{b}=$ Se basa en rangos negativos; $Z=$ resultados de la prueba de Wilcoxon al comparar relevancia y dominio de las competencias; Sig. = Significación; Estadísticamente significativas: ${ }^{*} p<, 05 * * p<, 01 * * * p<, 001$

Fuente: elaboración propia a partir del análisis de datos.

De otra parte, los análisis diferenciales informan lo siguiente. Atendiendo al sexo de los participantes (Cfr. Tabla 6), se hallan diferencias estadísticamente significativas respecto al dominio de las competencias "Conocer y comprender las bases teóricas y epistemológicas de los procesos y acciones formativas y educativas" (CDO1) y "Diseñar, ejecutar y evaluar planes de formación del profesorado, de formadores y de otros profesionales en diferentes contextos" (CDO6), siendo las mujeres las que presentan un mayor dominio ( $\mathrm{U}=795,00$; $p=, 004 ; \mathrm{U}=878,00 ; p=, 027)$. Por otra parte, también son las mujeres las que más consideran que han desarrollado la competencia "Asesorar en la toma de decisiones sobre problemas relevantes" (CDE10) frente a los hombres $(\mathrm{U}=895,00 ; p=, 036)$. 
Estudios Pedagógicos XLVII Nº 2: 197-213, 2021

VALORACIÓN Y EVALUACIÓN POR PARTE DE PEDAGOGOS EN FORMACIÓN RESPECTO DE LAS COMPETENCIAS ESPECÍFICAS DEL GRADO DE PEDAGOGÍA DE LA UNIVERSIDAD DE GRANADA

Tabla 6. Diferencias estadísticamente significativas

en la valoración de las competencias en función del sexo

\begin{tabular}{|l|c|c|c|c|c|c|c|c|}
\hline \multirow{2}{*}{ Competencias } & \multicolumn{3}{|c|}{ Hombre $(\boldsymbol{n = 2 2})$} & \multicolumn{3}{c|}{ Mujer $(\boldsymbol{n = 1 1 2})$} & \multicolumn{2}{c|}{ Mann-Whitney } \\
\cline { 2 - 9 } & $\boldsymbol{M}$ & $\boldsymbol{D T}$ & $\begin{array}{c}\text { Rango } \\
\text { promedio }\end{array}$ & $\boldsymbol{M}$ & $\boldsymbol{D} \boldsymbol{T}$ & $\begin{array}{c}\text { Rango } \\
\text { promedio }\end{array}$ & $\boldsymbol{U}$ & $\boldsymbol{P}$ \\
\hline CDO1 & 3,32 &, 57 & 47,64 & 3,87 &, 84 & 71,40 & 795,00 &, $004^{* *}$ \\
\hline CDO6 & 3,36 &, 80 & 54,41 & 3,90 & 1,16 & 70,66 & 878,00 &, $027^{*}$ \\
\hline CDE10 & 3,45 & 1,10 & 52,18 & 4,00 & 1,12 & 70,51 & 895,00 &, $036^{*}$ \\
\hline
\end{tabular}

Nota: $C D O=$ Dominio de la competencia; $C D E=$ Desarrollo de la competencia; $n=\mathrm{n}^{\circ}$ de elementos que compone la muestra; $M=$ Media; $D T=$ Desviación típica; $U=$ Resultados prueba U de Mann-Whitney; $p=$ Probabilidad asociada a U; Estadísticamente significativas: $* p<, 05 * * p<, 01 * * * p<, 001$

Fuente: elaboración propia a partir del análisis de datos.

Con respecto a la nota media obtenida por el alumnado (Cfr. Tabla 7), se encuentran diferencias estadísticamente significativas en relación con la relevancia de las competencias "Asesorar en la toma de decisiones sobre problemas relevantes (CRE10)" y "Conocer, analizar y atender a la diversidad social, educativa y cultural por razón de género, clase, etnia, edad, discapacidad, religión u otras (CRE11)", siendo el estudiante que ha obtenido sobresaliente el que otorga una mayor relevancia a dichas competencias $\left(\chi^{2}=5,51 ; p=, 039\right.$; $\left.\chi^{2}=6,20 ; p=, 045\right)$.

Tabla 7. Diferencias estadísticamente significativas en la valoración atendiendo a la nota media obtenida

\begin{tabular}{|c|c|c|c|c|c|c|c|c|c|c|c|c|}
\hline \multirow{3}{*}{ Competencias } & \multicolumn{9}{|c|}{ Nota media obtenida en el grado } & \multirow{2}{*}{\multicolumn{3}{|c|}{ Kruskal Wallis }} \\
\hline & \multicolumn{3}{|c|}{ Aprobado $(n=3)$} & \multicolumn{3}{|c|}{ Notable $(n=130)$} & \multicolumn{3}{|c|}{ Sobresaliente $(n=1)$} & & & \\
\hline & $M$ & $D T$ & $\begin{array}{c}\text { Rango } \\
\text { promedio }\end{array}$ & $M$ & $D T$ & $\begin{array}{c}\text { Rango } \\
\text { promedio }\end{array}$ & $M$ & $D T$ & \begin{tabular}{|c|} 
Rango \\
promedio
\end{tabular} & $\chi^{2}$ & $g l$ & $p$ \\
\hline & 3,67 &, 755 & 17,00 & 4,99 & 1,9 & 68,28 & 6,00 & 0 & 8 & 5,51 & 2 &, $039 *$ \\
\hline CRE11 & 4,00 & , 00 & 20,50 & 5,14 & ,97 & 68,29 & 6,00 & 0 & 105,50 & 6,20 & 2 &, $045^{*}$ \\
\hline
\end{tabular}

Nota: CRE = Relevancia de la competencia $; n=n^{o}$ de elementos que compone la muestra; $M=$ Media; DT = Desviación típica; $\chi^{2}=$ Estadístico asociado a la prueba Kruskal Wallis; $g l=$ Grados de libertad; $p=$ Probabilidad asociada a $\chi^{2}$; Estadísticamente significativas: $* p<, 05 * * p<, 01 * * * p<, 001$

Fuente: elaboración propia a partir del análisis de datos. 
En la misma línea del análisis anterior, se aprecian diferencias estadísticamente significativas en cuanto al dominio y el desarrollo de algunas competencias en función de la edad (Cfr. Tabla 8). Así, los estudiantes del Grupo 2 (22-23 años) ofrecen valoraciones superiores en cuanto al dominio de las competencias "Conocer y analizar las organizaciones, instituciones y sistemas de educación y formación como productos culturales e históricos y su influencia en la política y legislación educativa nacional e internacional" $\left(\chi^{2}=8,74 ; p\right.$ $=, 013)$ y "Realizar estudios prospectivos y evaluativos sobre características, necesidades y demandas pedagógicas" $\left(\chi^{2}=8,51 ; \mathrm{p}=, 014\right)$. A su vez, también es este grupo de edad el que considera haber desarrollado más la competencia "Realizar estudios prospectivos y evaluativos sobre características, necesidades y demandas pedagógicas", con respecto al resto $\left(\chi^{2}=7,72 ; p=, 021\right)$.

Tabla 8. Diferencias estadísticamente significativas en la valoración según el grupo de edad

\begin{tabular}{|c|c|c|c|c|c|c|c|c|c|c|c|c|}
\hline \multirow{3}{*}{ Competencias } & \multicolumn{9}{|c|}{ Edad } & \multirow{2}{*}{\multicolumn{3}{|c|}{ Kruskal Wallis }} \\
\hline & \multicolumn{3}{|c|}{ Grupo $1(n=67)$} & \multicolumn{3}{|c|}{ Grupo $2(n=46)$} & \multicolumn{3}{|c|}{ Grupo $3(n=21)$} & & & \\
\hline & $M$ & $D T$ & \begin{tabular}{|c|} 
Rango \\
promedio
\end{tabular} & $M$ & $D T$ & $\begin{array}{c}\text { Rango } \\
\text { promedio }\end{array}$ & $M$ & $D T$ & $\begin{array}{c}\text { Rango } \\
\text { promedio }\end{array}$ & $\chi^{2}$ & $g l$ & $p$ \\
\hline $\mathrm{CDO} 2$ & 3,61 & ,67 & 60,63 & 4,09 & ,98 & 80,03 & 3,71 & ,96 & 61,95 & 8,74 & 2 & ,013* \\
\hline $\mathrm{CDO} 13$ & 3,51 & 1,13 & 60,78 & 4,11 & 1,06 & 80,5 & 3,62 & ,98 & 60,45 & 8,51 & 2 & ,014* \\
\hline CDE13 & 3,90 & ,93 & 59,21 & 4,20 & 1,00 & 78,87 & 4,00 & ,90 & 69,05 & 7,72 & 2 &, $021 *$ \\
\hline
\end{tabular}

Nota: $C D O=$ Dominio de la competencia $; C D E=$ Desarrollo de la competencia $; n=\mathrm{n}^{\circ}$ de elementos que compone la muestra; $M=$ Media; $D T=$ Desviación típica; $\chi^{2}=$ Estadístico asociado a la prueba Kruskal Wallis; $g l$ $=$ Grados de libertad; $p=$ Probabilidad asociada a $\chi^{2}$; Estadísticamente significativas: $* p<, 05 * * p<, 01 * * * p<, 001$; Grupo 1 = 20-21 años; Grupo 2 = 22-23 años; Grupo $3=24$ años en adelante.

Fuente: elaboración propia a partir del análisis de datos.

\section{DISCUSIÓN Y CONCLUSIONES}

Partiendo del impacto que ha tenido el Plan Bolonia en todo el Espacio Europeo de Educación Superior y las propuestas que las universidades hacen de sus diferentes planes de estudios, la adaptación de los estudios superiores a las necesidades de la población implica proponer competencias y habilidades que capaciten a los estudiantes a ser competentes ante un mercado laboral en constante cambio (Magro, 2019). En este sentido, valorar dichas competencias supone el punto de partida para conocer cómo se están desenvolviendo las diferentes titulaciones, y, así, conseguir una educación de calidad.

Se ha podido apreciar como el alumnado de pedagogía manifestaba un mayor desarrollo de las competencias que el dominio declarado. Los estudiantes expresan la importancia de dichas competencias para su formación como pedagogos, pero existe un desequilibrio entre lo que se desarrolla en el plan de estudios y en el dominio que finalmente consiguen 
alcanzar. Del mismo modo, el grado de dominio no conseguía puntuaciones sobresalientes en las competencias planteadas. Ambos aspectos, se evidencian del mismo modo en la investigación desarrollada en el grado de Pedagogía de la Universidad de Murcia (González et al., 2015). Frente a una valoración de las competencias mejorable en cuanto a desarrollo y dominio, la relevancia otorgada a las mismas arroja puntuaciones superiores al resto.

En relación con las competencias de tipo social, aquellas que "incluyen motivaciones, valores, capacidad de relación en un contexto social organizativo" (ANECA, 2005, p. 155), resulta interesante resaltar que el alumnado no aporta puntuaciones altas, mostrándose medias y bajas en las competencias específicas C7, C10 y C11, vinculadas al diagnóstico, asesoramiento a personas o al análisis de la diversidad cultural. En contrapartida, en el trabajo de Díaz et al. (2016) se destacan buenas puntuaciones en competencias de este tipo vinculadas a aspectos afectivos y metacognitivos, arrojando resultados a favor de las mujeres. Sin embargo, la importancia otorgada a este tipo de competencias es notablemente superior al resto, evidenciando, del mismo modo, que la relevancia manifestada supera al desarrollo y dominio.

Parece curioso, como en una carrera vinculada íntimamente al contacto con personas, competencias de este tipo no se trabajen más en el aula ni se hayan dominado a lo largo de los tres-cuatro años de carrera. De esta forma, para una formación integral del alumno, no es óptima la presencia de debilidades de este tipo, ya que encarecen la valía del proceso de enseñanza-aprendizaje del experto en educación. En la misma línea, no se aprecian valoraciones sobresalientes en cuanto a la elaboración y diseño de proyectos, materiales educativos o programas de intervención, aspecto que sí obtenía buenas puntuaciones en el estudio realizado por Pérez et al. (2013), en cuanto al desarrollo y dominio.

Por otro lado, al igual que en estudios previos, Molero et al. (2010) y Martín et al. (2012), se encuentran diferencias estadísticamente significativas en cuanto al sexo, a favor de las mujeres. Aunque estos datos, deben tomarse con cautela, pues la mayoría de estudiantes son mujeres, presentándose una minoría de hombres, y no se cuenta con una muestra balanceada por sexo.

En cuanto a la posible influencia de las calificaciones, se observa que, a mayor nota media, más relevancia les otorgan a las competencias. Al igual que con los resultados anteriores, se debe tener en cuenta que el grueso del estudiantado se ubica en el notable y, por tanto, sería interesante bien ampliar la muestra para obtener mayor diversidad o modificar el dato sociodemográfico de la calificación, que venía establecido como aprobado, notable o sobresaliente, y dejarlo abierto para que alumno indique su nota media numérica.

Atendiendo a la edad, al igual que en otros estudios, como los de Díaz et al. (2016) y Surdez et al. (2018), se aprecian diferencias estadísticamente significativas, a favor del grupo 2 (22-23 años). Así, se aprecia un aumento en el grado de desarrollo y dominio con la edad.

Tras lo planteado con anterioridad, se observa como los estudiantes manifiestan la importancia que las competencias tienen sobre su formación como pedagogos. En contraposición, se aprecia como el dominio de las mismas de cara a una futura profesión es menor, pese a la importancia que les conceden como especialistas de la educación. En esta línea, resultan relevantes las palabras de González et al. (2015) "en un modelo de aprendizaje basado en competencias, el profesor debe valorar la diversidad de alternativas metodológicas, más aún, cuando se trata de competencias específicas, encargadas de definir el perfil profesional a partir de conocimientos, habilidades, aptitudes y valores" ( $\mathrm{p}$. 
409). El alumnado es consciente de que un enfoque más comprensivo y relacionado con las problemáticas actuales a las que se enfrenta el mercado laboral actual es necesario, por lo que es imprescindible un grado universitario que favorezca el desarrollo de habilidades específicas para los diversos puestos en los que el pedagogo pueda intervenir y desenvolverse profesionalmente.

Sería deseable que las competencias no oscilaran en valores medios-bajos de dominio, sino que se situaran por encima. A su vez, es preciso plantear en qué medida se desarrollan dichas competencias en las distintas materias del grado, ya que, pese a existir un esquema de qué competencias se exponen en las diferentes asignaturas, el alumnado no declara desarrollarlas ni dominarlas tanto como desearía. Como propone Rodríguez et al. (2018) sería enriquecedor ofrecer "a los estudiantes universitarios una formación sostenible en coherencia con el contexto actual” (p. 200).

\section{REFERENCIAS BIBLIOGRÁFICAS}

Aguilar, J. M., Alías, A., Álvarez, J., Fernández, J. M., Pérez, E. y Hernández, A. I. (2015). Necesidades de formación del profesor universitario en competencias relacionadas con la acción tutorial. Revista de Docencia Universitaria (REDU), 13(3), 357-375. https://dialnet.unirioja.es/ servlet/articulo?codigo $=5300724$

Alonso-Martín, P. (2010). La valoración de la importancia de las competencias transversales: comparación de su percepción al inicio y final de curso en alumnos de Psicología. Revista de Investigación Educativa, 28(1), 119-140. http://revistas.um.es/rie/article/view/97821

ANECA (2005). Libro blanco. Título de Grado en Pedagogía y Educación Social. Omán Impresores. http://www.aneca.es/Documentos-y-publicaciones/Libros-Blancos

Caurcel, M. J., Rodríguez Fuentes, A., García Guzmán, A. y Ramos García, A. (2008). Analysis of New Roles and Requirements for Lecturers and Students According to the European Higher Education Area. The International Journal of Learning, 14(11), 27-34. http://dx.doi. org/10.18848/1447-9494/CGP/v14i11/45516

Castillo, S. y Cabrerizo, J. (2010). Evaluación educativa de aprendizajes y competencias. Pearson Educación.

Coiduras, J., París, G., Torrelles, C. y Carrera, X. (2018). La evaluación de competencias en una experiencia de formación dual de maestros: diferencias y semejanzas entre tutores de escuela y de universidad. Estudios Pedagógicos, 40(Especial), 29-48. http://dx.doi.org/10.4067/S071807052014000200003

Díaz, I., Cebrián, S. y Fuster, I. (2016). Las competencias en TIC de estudiantes universitarios del ámbito de la educación y su relación con las estrategias de aprendizaje. RELIEVE, 22(1), 1-24. https://doi.org/10.7203/relieve.22.1.8159

Domingo, J., Gallego, J. L., García, I. y Rodríguez, A. (2010). Competencias comunicativas del maestro en formación. Profesorado. Profesorado. Revista sobre currículum y formación del profesorado, 14(2), 304-323. http://www.ugr.es/ recfpro/rev142COL7.pdf

Gallego, J. L. y Rodríguez, A. (2015). Competencias comunicativas de maestros en formación de educación especial. Educación y Educadores, 18(2), 209-225. http://dx.doi.org/10.5294/ edu.2015.18.2.2

García, D. y Bernabé, M. ${ }^{a}$ M. (2019). Formación y preparación musical en el Grado de Educación Infantil: Consideraciones de las alumnas en Educación Superior. Revista Prisma Social, 25, 126178. http://revistaprismasocial.es/article/view/2692

García, J., Guzmán, A. y Murillo, G. (2014). Evaluación de competencias y módulos en un currículo innovador. El caso de la licenciatura en Diseño y Desarrollo de Espacios Educativos con TIC 
Estudios Pedagógicos XLVII N 2: 197-213, 2021

VALORACIÓN Y EVALUACIÓN POR PARTE DE PEDAGOGOS EN FORMACIÓN RESPECTO DE LAS COMPETENCIAS ESPECÍFICAS DEL GRADO DE PEDAGOGÍA DE LA UNIVERSIDAD DE GRANADA

de la Universidad de Costa Rica. Perfiles Educativos, 36(143), 67-85. https://doi.org/10.1016/ S0185-2698(14)70610-5

García, J., Lorenzo, M. y Vázquez, A. (2019). Diseño y validación de una escala de competencias específicas para graduados en Pedagogía (ECEG-P). Revista Española de Orientación y Psicopedagogía, 30(3), 84-101. https://www.researchgate.net/publication/338792101_ diseno_y_validacion_de_una_escala_de_competencias_especificas_para_graduados_en_ pedagogia_eceg-p_design_and_validation_of_the_specific_competences_for_graduates_in_ pedagogy_scale_eceg-p

González, C. y Martínez, P. (2015). Competencias transversales en educación superior: un estudio en la Universidad de Murcia (España) y la Universidad Católica de Córdoba (Argentina). En AIDIPE (Ed.), Investigar con y para la sociedad (pp. 833-844). Asociación Interuniversitaria de Investigación Pedagógica (AIDIPE). https://www.uv.es/aidipe/congresos/Actas_XVI_ Congreso.pdf

González, C., Martínez, P. y González, N. (2015). El perfil formativo del graduado en Pedagogía: La visión del alumnado. Profesorado. Revista de currículum y formación del profesorado, 19(1), 394- 412. http://www.redalyc.org/articulo.oa?id=56738729024

Flores-Lueg, C. y Roig, R. (2018). Percepción de estudiantes de Pedagogía sobre el desarrollo de su competencia digital a lo largo de su proceso formativo. Estudios Pedagógicos, 42(3), 129-148. http://dx.doi.org/10.4067/S0718-07052016000400007

López, C., Benedito, V. y León, M. J. (2016). El Enfoque de Competencias en la Formación Universitaria y su Impacto en la Evaluación. La Perspectiva de un Grupo de Profesionales Expertos en Pedagogía. Formación Universitaria, 9(4), 11-22. http://dx.doi.org/10.4067/S071850062016000400003

Makuc, M. (2018). Teorías implícitas sobre comprensión textual y la competencia lectora de estudiantes de primer año de la Universidad de Magallanes. Estudios Pedagógicos, 37(1), $237-$ 254. http://dx.doi.org/10.4067/S0718-07052011000100013

Magro, M. (2019). Necesidades formativas del profesorado de educación infantil para la atención a la diversidad de colegios rurales agrupados. Revista Prisma Social, 25, 103-125. https:// revistaprismasocial.es/article/view/2683

Martí, J. J., Martí, M., Vargas, O. y Moncayo, J. E. (2018). Reflexión sobre los discursos en educación superior, una mirada desde la psicología social crítica. Revista de la Educación Superior, 32(172), 33-55. http://www.scielo.org.mx/scielo.php?pid=S0185-27602014000400003\&script=sci_abstract

Martín, M. L., Díaz, E. y Del Barrio, L. (2012). Metodología docente y evaluación por competencias: una experiencia en la materia Dirección de Producción. Investigaciones Europeas de Dirección y Economía de la Empresa, 18(3), 237-247. https://doi.org/10.1016/j.iedee.2012.05.003

Molero, D., Ortega, F. y Moreno, M. R. (2010). Diferencias en la adquisición de competencias emocionales en función del género. Revista Electrónica de Investigación y Docencia (REID), 3, 165-172. https://revistaselectronicas.ujaen.es/index.php/reid/article/view/1167/989

Montero, I. y León O. G. (2007). A guide for naming research studies in Psychology. International Journal of Clinical and Health Psychology, 7(3), 847-862. http://www.aepc.es/ijchp/articulos. php?coid=Espa\%EF\%BF\%BDol\&id=256

Organización para la Cooperación y el Desarrollo Económico (OCDE) (2005). La definición y selección de competencias clave. Resumen ejecutivo. OCDE.

Pérez, C. (2005). Muestreo estadístico: conceptos y problemas resueltos. Pearson Educación.

Pérez, J., Martínez, M., González, C., González, N. y Martínez, P. (2013). Valoración de las competencias del Grado de Educación Social: La visión del alumnado. En C. Cardona, E. Chiner y A. Giner (Eds.), II Internacional Modelos de Investigación Educativa de la Asociación Interuniversitaria de Investigación Pedagógica (AIDIPE). Actas del XVI Congreso Nacional (pp. 381-389). Asociación Interuniversitaria de Investigación Pedagógica (AIDIPE). https:// www.uv.es/aidipe/congresos/Actas_XVI_Congreso.pdf 
Pérez, M., Miguel, A. E., Moreno, J. y Martínez, K. A. (2019). Educación media superior y desarrollo sustentable en las ciudades del estado de Oaxaca, México. Perfiles Educativos, 41(163), 59-74. https://doi.org/10.22201/iisue.24486167e.2019.163.58824

Reche, E., Martín, M. A. y González, I. (2019). Autopercepción de la adquisición de las competencias informacional y comunicativa para la elaboración de trabajos académicos en la universidad. Perfiles Educativos, 41(165), 131-146. https://doi.org/10.22201/iisue.24486167e.2019.165.59170

Richart, R. E., Álvarez, E. P. y Martínez, R. C. (2019). Competencias del perfil del administrador. Análisis comparativo en diferentes modalidades educativas. Perfiles Educativos, 41(164), 82-98. https://doi.org/10.22201/iisue.24486167e.2019.164.59108

Rodríguez, H. (2007). El paradigma de las competencias hacia la educación superior. Revista Facultad de Ciencias Económicas: Investigación y Reflexión, 15(1), 145-165. https://www. redalyc.org/pdf/909/90915108.pdf

Rodríguez, G., Ibarra, M. S. y Cubero, J. (2018). Competencias básicas relacionadas con la evaluación. Un estudio sobre la percepción de los estudiantes universitarios. Educación XXI, 21(1), 181-208. https://www.redalyc.org/pdf/706/70653466009.pdf

Romero, M. y Faouzi, T. (2018). Validación de un modelo de competencias pedagógicas para docentes de Educación Media Técnica. Educación y Educadores, 21(1), 114-132. http://dx.doi. org/10.5294/edu.2018.21.1.6

Ruiz, J. M. (2010). Evaluación del diseño de una asignatura por competencias, dentro del EEES, en la carrera de pedagogía. Estudio de un caso real. Revista de Educación, 351, 435-460. https:// www.educacionyfp.gob.es/dam/jcr:df131819-947c-42cc-a789-6de902b93b7c/re35118-pdf.pdf

Serrano, R. y Pontes, A. (2015). Nivel de desarrollo de las competencias y objetivos generales del Máster Formación del Profesorado de Enseñanza Secundaria. Perfiles Educativos, 37(150), 39-55. http://www.scielo.org.mx/scielo.php?script=sci_abstract\&pid=S0185-26982015000400003\&ln $\mathrm{g}=\mathrm{es} \& \mathrm{nrm}=\mathrm{iso}$

Sistema integral de gestión de alumnos (SIGA) (2017-2018). Listado del número de alumnos matriculado en asignaturas de $3^{\circ}$ y $4^{\circ}$ del Grado de Pedagogía durante el curso académico 2017-2018. Secretaria de la Facultad de Ciencias de la Educación de la Universidad de Granada.

Surdez, E., Sandoval, M. y Lamoyi, C. (2018). Satisfacción estudiantil en la valoración de la calidad educativa universitaria. Educación y Educadores, 21(1), 9-26. http://dx.doi.org/10.5294/ edu.2018.21.1.1

Universidad de Granada (2017). Memoria académica de la Facultad de Ciencias de la Educación (curso 2017-18). Secretaria General. http://educacion.ugr.es/pages/facultad/documentos/ memoriaacademica201718

Velásquez, M., Cornejo, C. y Roco, Á. (2018). Evaluación de la competencia lectora en estudiantes de primer año de carreras del área humanista y carreras del área de la salud en tres universidades del consejo de rectores. Estudios Pedagógicos, 3(1), 123-138. http://dx.doi.org/10.4067/S071807052008000100007 
